\title{
Enhanced speed advice for connected vehicles in the proximity of signalized intersections
}

\author{
Evangelos Mintsis $^{1 *} \mathbb{D}$, Eleni I. Vlahogianni ${ }^{1}$, Evangelos Mitsakis ${ }^{2}$ and Seckin Ozkul ${ }^{3}$
}

\begin{abstract}
Technological advancements in the field of transportation are gradually enabling cooperative, connected and automated mobility (CCAM). The progress in information and communication technology (ICT) has provided mature solutions for infrastructure-to-vehicle (I2V) communication, which enables the deployment of CooperativeITS (C-ITS) services that can foster comfortable, safe, environmentally friendly, and more efficient traffic operations. This study focuses on the enhancement of speed advice comfort and safety in the proximity of signalized intersections, while ensuring energy and traffic efficiency. A detailed microscopic simulation model of an urban network in the city of Thessaloniki, Greece is used as test bed. The performance of dynamic eco-driving is evaluated for different penetration rates of the dynamic eco-driving technology and varying traffic conditions. The simulation analysis indicates that speed advice can be comfortable and safe without adversely impacting energy and traffic efficiency. However, efficient deployment of dynamic eco-driving depends on road design characteristics, activation distance of the service, traffic signal plans, and prevailing traffic conditions.
\end{abstract}

Keywords: Dynamic eco-driving, Enhanced speed advice, Connected vehicle, Microscopic traffic simulation, Signalized intersection, Greenhouse gas emissions

\section{Introduction}

C-ITS and Advanced Driver Assistance Systems (ADAS) can significantly enhance comfort, safety, traffic efficiency and energy savings via real-time information provision (tailored to the needs of individual vehicles), and cooperative driving [30]. Road safety impact assessment of the latter systems has been conducted through real world experiments [26] and with the use of microscopic traffic simulation tools [31] that utilize surrogate measures of safety to indicate conflict risk for both uninterrupted and interrupted traffic flow [3, 9, 10, 12, 22].

C-ITS applications and ADAS that enable eco-driving and yield environmental benefits have also received significant attention from funding agencies, vehicle manufacturers, road authorities, technology providers, and the research community. Specifically, dynamic eco-driving in the proximity of signalized intersection uses real-time traffic and Signal Phase and Timing (SPaT) information to communicate robust and real-time speed and/or countdown advice to connected vehicles (CVs). Comprehensive reviews discussing different aspects of dynamic ecodriving in a connected (and automated) road environment can be found in $[2,13,16,29,45,47,49]$.

Methodologically, the operation and performance of dynamic eco-driving was previously assessed through microscopic traffic simulation studies, driving simulators, controlled field experiments and real-world experiments [29].

\footnotetext{
*Correspondence: vmintsis@gmail.com

'Department of Transportation Planning and Engineering, School of Civil Engineering, National Technical University of Athens, 5 Iroon Polytechniou Str., Zografou Campus, 15773 Athens, Greece

Full list of author information is available at the end of the article
} 
The operation of most dynamic eco-driving models relies on the assumption that traffic signals are pre-timed [5, 20, 27, 40, 41, 50, 55]. However, researchers developed and simulated methodologies that facilitate the implementation of dynamic eco-driving services for actuated and adaptive traffic lights using empirical signal and loop detector data $[6,14,25,32,44,53]$. Erdmann and Liang et al. [11, 23] proposed the combination of adaptive signal control with Green-Light-OptimalSpeed-Advisory (GLOSA) to develop signal plans that exploit vehicle state information.

Efforts were also placed for the development of dynamic eco-driving models that can estimate energy and traffic efficient speed advice for $\mathrm{CV}$ platoons in the proximity of signalized intersections [7, 43, 52, 54, 59]. Recently, artificial intelligence has been also used for applying dynamic eco-driving control in the proximity of signalized intersections $[33,58]$.

The majority of proposed dynamic eco-driving models that were evaluated with the use of microscopic traffic simulation tools considered hypothetical networks with simplified demand scenarios [5, 18, 27, 41, 42, 55, 56]. A few studies focused on real-world isolated signalized intersections [21, 48], and others simulated either single vehicle [15] or multi vehicle $[4,46,51]$ scenarios along actual urban arterial corridors (very limited information is provided though regarding the calibration and validation of the respective real-world simulation models). Moreover, deceleration strategies received greater interest, since they provide higher energy savings potential, while some researchers considered the recommendation of acceleration as safety critical [41]. Finally, focus has been also placed in modelling and simulation of human factors related to dynamic eco-driving [24, 38, 40, 57].

The literature review indicates that limited focus was previously placed on the comfort and safety of dynamic eco-driving technologies [29]. Early evidence from field testing of an eco-cruise control system in the vicinity of traffic signalized intersections showed that manual speed adaptation based on countdown advice proved less comfortable, but equally safe and desirable compared to automated eco-cooperative adaptive cruise control (EcoCACC) [39]. Thus, there is significant potential for enhancing dynamic eco-driving performance via the introduction of novel features that improve comfort, user acceptance and safety.

Undoubtedly, drivers/passengers would be more willing to adopt dynamic eco-driving if it ensured comfortable, safe and intuitive speed advice. According to the profile of existing deceleration strategies, a CV initially decelerates and subsequently cruises at a steady-state speed towards a signalized intersection until the signal status changes to green, when vehicle accelerates back to its desired speed beyond the signalized intersection. This implies that existing dynamic eco-driving services instruct CVs to cruise at significant steady speed while the vehicle approaches the signalized intersection and the signal status remains red. In this case, many drivers/passengers would feel uncomfortable driving/riding a vehicle that cruises in close vicinity to a signalized intersection while the traffic light status is still red. That would be especially true in the early stages of CVs market introduction when mixed traffic conditions are expected to prevail on the streets and drivers/passengers will be less familiar with CV technology.

This study proposes and evaluates enhancements on an existing dynamic eco-driving model (velocity planning algorithm - VPA) that encompass the following novel features:

- provision of non-crawling speed advice, and

- vehicle acceleration begins prior to CV arrival at signalized intersection after deceleration strategy

The enhanced VPA is examined with the use of microscopic traffic simulation along an actual urban arterial corridor that was thoroughly calibrated against real traffic conditions.

\section{Enhanced speed advice}

Enhanced dynamic eco-driving accounts for intuitive speed advice that drivers/passengers can easily and conveniently adapt to, and encompasses comfortable accelerations/decelerations, acceptable cruising speeds, as well as guidance that facilitates safe interactions with surrounding road users and elements (e.g. traffic lights). As mentioned above, existing literature has overlooked specific aspects of speed advice pertaining to comfort and safety which this study aims to address. To this end, we present in the following sections the reference model (VPA) previously developed by [55] and an enhanced VPA version proposed by this study that promotes speed advice comfort and safety without adversely impacting energy and traffic efficiency.

\subsection{Velocity planning algorithm (VPA)}

Xia et al. [55] introduced VPA considering that energy savings can be realized when drivers exhibit the following behavior:

- maintain a steady-state speed near the speed limit,

- keep a safe headway distance from the leading vehicle, and

- avoid idling, or idle the least possible time at the traffic light if this is unavoidable.

Thus, an optimization problem was formulated that minimized a vehicle's tractive force and idling time while 
accounting for ride comfort and the local speed limit $\left(v_{\text {lim }}\right)$. To avoid stopping at a traffic light, a vehicle should arrive at the signalized intersection during a green signal status. Based on the current signal status, a green arrival interval can be estimated as:

$$
t_{\text {arrival }}= \begin{cases}{\left[0, t_{r}\right\} \text { or }\left[t_{g}, t_{r 1}\right),} & \text { if signal status }=\text { green } \\ {\left[t_{g}, t_{r}\right),} & \text { if signal status }=\text { red }\end{cases}
$$

where $t_{r}$ is the time to the upcoming red phase, $t_{g}$ represents the time to the next green phase, and $t_{r 1}$ is the time to the second red phase. Thus, if the signal is green, a vehicle can either cruise at current speed or accelerate to a target speed to pass through the intersection during the first green window or decelerate and cross the intersection during the second green window. If the signal is red (yellow time is considered to be red time), the vehicle can cruise at current speed or decelerate to a target speed to cross the intersection during the upcoming green window.

The possible values of $t_{\text {arrival }}$ can range between $\left[t_{l}, t_{h}\right]$, where $t_{l}$ and $t_{h}$ are low and high values according to Eq. 1 . Given the range $\left[t_{l}, t_{h}\right]$ and the vehicle's distance to intersection $d_{i n t}$, the possible target velocities $v_{\text {arrival }}$ can be expressed as the range $\left[v_{l}, v_{h}\right]$, where $v_{l}$ is the maximum between zero and $v_{l o}\left(v_{l o}=d_{\text {int }} / t_{h}\right)$ and $v_{h}$ is the minimum between $v_{\text {lim }}$ and $v_{h o}\left(v_{h o}=d_{\text {int }} / t_{l}\right)$. Evidently, $d_{\text {int }}$ and signal timing information are key parameters for the estimation of optimal speed trajectories.

When $v_{\text {arrival }}$ is estimated, the provision of speed advice to $\mathrm{CV}$ is determined according to its current speed $v_{c}$. If $v_{c}$ lies within $\left[v_{l}, v_{h}\right]$, then the vehicle can pass the intersection cruising at current speed. Alternatively, it can accelerate or decelerate with respect to $v_{h}$, which [55] have selected as the target velocity to achieve travel time savings apart from environmental benefits. The energy-efficient speed profiles are estimated according to the following functions:

$$
v_{\text {opt }}= \begin{cases}v_{h}-v_{d} * \cos (\mu t), & \text { for } 0 \leq t<\frac{\pi}{2 \mu} \\ v_{h}-v_{d} * \frac{\mu}{\rho} * \cos \left(t-\frac{\pi}{2 \mu}+\frac{\pi}{2 \rho}\right), & \text { for } \frac{\pi}{2 \mu} \leq t<\left(\frac{\pi}{2 \rho}+\frac{\pi}{2 \mu}\right) \\ v_{h}+v_{d} * \frac{\mu}{\rho} & \text { for }\left(\frac{\pi}{2 \rho}+\frac{\pi}{2 \mu}\right) \leq t \leq \frac{d_{\text {int }}}{v_{h}}\end{cases}
$$

where $v_{d}$ is equal to $v_{h}-v_{c}$. Positive $v_{d}$ values generate acceleration profiles, and negative values generate deceleration profiles. The only unknown parameters in Eq. 2 are $\mu$ and $\rho$, which determine the acceleration/deceleration rate. The higher the value of $\mu$, the higher the acceleration/deceleration rate. The values of $\mu$ and $\rho$ can be computed by solving the following three constraints:

$$
\left\{\begin{array}{l}
\int_{0}^{\frac{\pi}{2 \mu}}\left(v_{h}-v_{d} * \cos (\mu t)\right) d t+\int_{\frac{\pi}{2 \mu}}^{\frac{\pi}{2 \rho}+\frac{\pi}{2 \mu}}\left(v_{h}-v_{d} * \frac{\mu}{\rho} * \cos \rho\left(t-\frac{\pi}{2 \rho}+\frac{\pi}{2 \mu}\right)\right) d t \\
+\left(v_{h}+v_{d} * \frac{\mu}{\rho}\right) *\left(\frac{d_{i n t}}{v_{h}}-\frac{\pi}{2 \rho}-\frac{\pi}{2 \mu}\right)=d_{\text {int }} \\
j \operatorname{jerk} k_{\max }=\left|v_{d} * \mu * \rho\right| \leq 10 \text { and } a_{\max } \leq 2.5 \mathrm{~m} / \mathrm{s}^{2} \\
\mu=\max \{\mu\}
\end{array}\right.
$$

The first constraint in Eq. 3 is the distance constraint, which ensures vehicle's arrival at the downstream signalized intersection in the shortest time. The second constraint pertains to ride comfort. The third was set based on the finding of [55], which suggests that minimization of fuel consumption and emissions occurs for the largest possible $\mu$ value (i.e. a vehicle accelerates sharply instead of smoothly to $v_{h}$ ). Moreover, it has to be noted that VPA can be explicitly implemented at signalized intersections with fixed signal control plans, and it does not consider queue dynamics at signalized intersections. A more detailed description of VPA can be found in [55].

\subsection{Enhanced velocity planning algorithm (EVPA)}

This study introduced enhancements to the control logic of the reference model (VPA) accounting for actual behavioral traits of drivers. The enhanced velocity planning algorithm (EVPA) increases the comfort and safety of the provided speed advice to facilitate acceptance of dynamic eco-driving service from the driver's/passenger's side.

The control logic of the reference model implies that the minimum speed advice is an explicit function of the vehicle's traveling state (approach speed and distance to the signalized intersection) and the signal timing information of the signalized intersection. Thus, $v_{l}$ could acquire rather low values (e.g. $10 \mathrm{~km} / \mathrm{h}$ ), which implies that a vehicle might be advised to cruise towards a signalized intersection at a crawling speed. However, in practice, drivers would refrain from driving below a minimum speed threshold (anxiety reasons), irrespective of the provided speed advice. Thus, the authors propose that $v_{\text {arrival }}$ is not only bounded on the upper limit by the speed limit, but also on the lower limit by a minimum acceptable speed value $\left(v_{\min }\right)$. Therefore, $v_{l}$ would become the maximum between $v_{\min }$ and $v_{l o}\left(v_{l o}=d_{i n t} / t_{h}\right)$. It is expected that this enhancement will increase the indirect benefits of dynamic eco-driving, since legacy vehicles (LVs) will overtake CVs less frequently, thus inducing less turbulence to traffic. Additionally, previous research has shown that cruising at low speeds at the end of deceleration strategies might incur higher energy 
consumption, even compared to a standstill strategy [41].

The second enhancement also pertains to deceleration strategies. According to the control logic of existing dynamic eco-driving models, a CV's arrival at traffic lights after the implementation of a deceleration strategy is concurrent with the onset of the green phase. However, many drivers/passengers would feel uncomfortable riding a vehicle that cruises at high steady speed in close vicinity to a signalized intersection while the signal status is still red. Therefore, this study suggests that the lowest cruising speed $v_{c r}$ of the initially estimated deceleration profile is used for the computation of the CV's practical stopping distance, assuming it had complied with the initial deceleration strategy. In this case, the vehicle's practical stopping distance $d_{\text {stop }}$ is given by the following formula:

$$
d_{\text {stop }}=\frac{v_{c r}^{2}}{2 g\left(\left(\frac{a_{d}}{g}\right) \pm G\right)}
$$

where $a_{d}$ is the deceleration rate, $g$ is the gravitational constant, and $G$ is the roadway grade. Eq. 4 provides an estimate of typical braking distances and is more simplistic and usable than the theoretical stopping distance one. Given the assumption that CVs fully stop and road grades are small, mass factor accounting for moments of inertia during braking (which is considered for the estimation of theoretical stopping distance) can be ignored due to its small effects [28]. Moreover, we assume that friction is always guaranteed in our simulation experiments and anomalous situations such as sudden and strong braking do not occur.

Subsequently, the practical stopping distance is subtracted from $d_{i n t}$, and the result $\left(d^{\prime}=d_{i n t}-d_{\text {stop }}\right)$ is returned to the algorithm for the estimation of an enhanced deceleration profile. According to this updated deceleration profile, the vehicle decelerates to a lower cruising speed $v_{c r}^{\prime}$ compared to the initial one, but the onset of the green phase occurs prior to the vehicle's arrival at the signalized intersection. Moreover, sufficient time and space remain available for the CV to stop in case of red light running from the opposite direction. Since the practical stopping distance is a function of the vehicle's cruising speed, the EVPA is expected to perform efficiently within a wide range of cruising speeds. The enhanced dynamic eco-driving service is expected to be perceived as more intuitive, convenient and safer by drivers, who would thus increase their confidence regarding the system's operation and performance.

\section{Simulation experiment}

\subsection{Microscopic traffic simulation model}

A detailed microscopic simulation model of an urban arterial corridor in the city of Thessaloniki, Greece, was developed with the use of the microscopic traffic simulation tool Aimsun. Its total length is $15 \mathrm{~km}$ (road grade is nearly zero across the full length of the corridor) and it encompasses 26 signalized intersections (17 equipped with road-side units) which are controlled by pre-timed signal control plans. The reference and the enhanced dynamic eco-driving services were deployed on 23 signalized intersection approaches (IA) (highlighted in yellow) of the examined simulation network (Fig. 1). Side-street parking and seven public transport lines (along with their corresponding time plans) that travesrse the central business district (CBD) of Thessaloniki were simulated as well.

A thorough macroscopic calibration process was conducted to ensure the ability of the microscopic traffic simulation model to replicate actual traffic operations (without dynamic eco-driving service) on the examined road network. Calibration parameters of Aimsun driver models (car-following, lane-changing, and gapacceptance models) were adjusted for the reconciliation of field and simulated traffic counts. Field traffic data were obtained from several traffic detectors that monitor traffic conditions in the CBD of Thessaloniki. The latter data contain traffic volumes, average time mean speed, and travel time information for selected network routes.

Field and simulated traffic counts were used for the conduct of the appropriate statistical test (GEH) to verify the validity of the simulation model [8]. The estimated GEH values were lower than 5 for more than $85 \%$ of the selected detector stations (volume and speed counts). Moreover, GEH index was also lower than 5 when comparing average travel time between field and simulation along the urban arterial corridor shown in Fig. 1. Thus, the calibration procedure demonstrated that the simulation model can credibly replicate traffic operations pertaining to manual driving on the test network.

However, we also deem that our simulation model remains valid for different market penetration rates of dynamic eco-driving technology, since we assumed that $\mathrm{CVs}$ are manually driven beyond the service activation zone (cf. Section 3.2) and existing literature [2, 13, 16, $29,45,47,49$ ] addressing the impacts of dynamic ecodriving on traffic operations does not indicate changes to route choice due to speed advice provision in the proximity of signalized intersections.

\section{2 (E)VPA - Application Programming Interface (API)}

VPA and EVPA were simulated in Aimsun with the use of an Application Programming Interface (API) that was directly interfaced with the core Aimsun models. The 


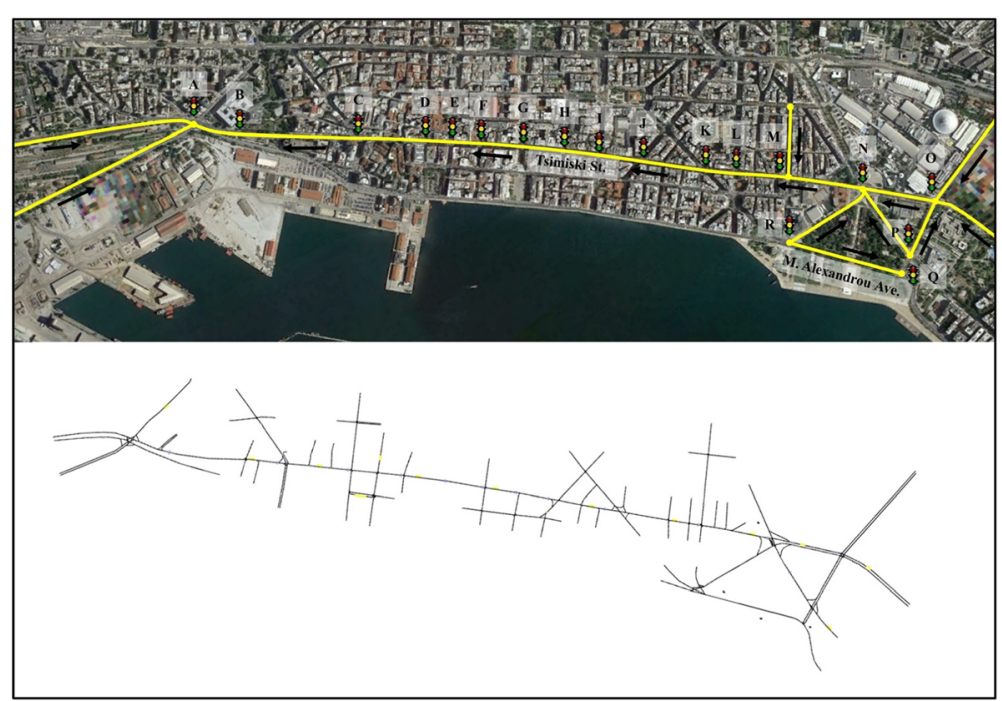

Fig. 1 Test site in Thessaloniki, Greece (real world and simulation)

API estimates a single energy optimal driving strategy for every $\mathrm{CV}$ that enters the dynamic eco-driving service activation zone. Then, the CV becomes "tracked" in the simulation and strictly follows the provided speed advice (every simulation time step) until it crosses the signalized intersection. Thus, CVs fully comply with the estimated speed advice within the service activation zone. However, a CV can discard speed advice if it enters carfollowing state. An empirical formula was used to assess the car-following state of CVs during the simulation [37]. The maximum car-following distance is given as:

$$
x_{c f}=T u_{k-1}+\beta
$$

where $T$ is a time constant, $u_{k-1}$ is the speed of the following vehicle, and $\beta$ is the average distance between two vehicles in standstill. If a CV's distance to the leader becomes shorter than $x_{c f}$, then it becomes "untracked" in the simulation and its motion is subsequently dictated by the Aimsun driver models. In this case, an updated speed advice is not provided to the CV even though it is still driving within the activation zone. The behavior of $\mathrm{CV}$ beyond the activation zone is determined by Aimsun driver models that are parametrized to reflect manual driving conditions.

The length of the activation zone (per signalized IA) is set equal to the total length of the corresponding signalized IA (road section between two consecutive intersections). During the simulation of VPA, the estimated speed advice can range between 5 and $50 \mathrm{~km} / \mathrm{h}$. On the other hand, while EVPA is simulated the estimated speed advice can range between the minimum cruising speed after deceleration $(20 \mathrm{~km} / \mathrm{h})$ and the speed limit
$(50 \mathrm{~km} / \mathrm{h})$. Table 1 provides an elaborate list of the parameter values that affect the operation of the reference and enhanced models in the simulation experiments.

\subsection{Microscopic emission model}

To estimate carbon dioxide $\left(\mathrm{CO}_{2}\right)$ emissions within the simulation loop (second-by-second estimation, $1 \mathrm{~Hz}$.), the Panis microscopic emission model calibrated with real world emission data is used [36]. As this model combines multiple non-linear regression models to estimate emission functions per vehicle type and pollutant (with instantaneous speed and acceleration as explanatory variables) it was considered relevant for the evaluation of the environmental impacts of dynamic ecodriving.

The fleet composition with respect to engine type for Greece was obtained from [1]. To this end, in our simulation experiments taxis, heavy duty vehicles (HDV), and buses run on diesel engines. Passenger cars are divided into the following shares according to their fuel type: 92\% petrol, 5\% diesel, and 2\% LPG. The emission

Table 1 Parameter values used in the simulation experiments

\begin{tabular}{lll}
\hline Variables & Description & Value(s) \\
\hline$V_{\text {lim }}$ & Speed limit $(\mathrm{km} / \mathrm{h})$ & 50 \\
$\mu$ & Acceleration rate parameter $(\mathrm{m} / \mathrm{s} \wedge 2)$ & 0.15 \\
$V_{\text {min }}$ & Minimum cruising speed after deceleration $(\mathrm{km} / \mathrm{h})$ & 20 \\
$g$ & Gravitational constant $(\mathrm{m} / \mathrm{s} \wedge 2)$ & 9.807 \\
$a_{d}$ & Normal deceleration rate $(\mathrm{m} / \mathrm{s} \wedge 2)$ & 4.00 \\
$G$ & Road grade $(\%)$ & 0 \\
$T$ & Time constant (sec) & 1.02 \\
$\beta$ & Average headway distance in standstill (meters) & 3.5 \\
\hline
\end{tabular}


constants used for the estimation of $\mathrm{CO}_{2}$ emissions per combination of vehicle and engine type are presented in Table 2.

\subsection{Simulation scenarios}

The performance of the reference (VPA) and the proposed (EVPA) speed advice algorithms was assessed for different traffic demand levels and different penetration rates of the dynamic eco-driving technology (Table 3). In total, 48 scenarios were simulated (38 with service on and 10 with service off). The calibration scenario corresponds to D100 traffic demand level (initial demand input to the microscopic simulation model). The effect of the penetration rate of the $\mathrm{CV}$ technology was tested both for uncongested (D50) and congested (D100) traffic conditions. On the other hand, the performance of $\mathrm{CV}$ technology for a wide spectrum of traffic conditions (uncongested - near congested - congested/D10 D100) was evaluated for three different penetration rates (low - moderate - high/P15 - P50 - P100). Speed advice was explicitly provided to passenger cars and taxis among the simulated vehicle types (passenger cars, taxis, trucks, and buses), since the reference model was explicitly developed for light-duty vehicles.

Aimsun is a stochastic microscopic traffic simulation tool. Thus, multiple runs of each simulated scenario were executed so that the obtained simulation outputs can be statistically significant. Initially, five runs of the calibration scenario were executed (each corresponding to a different random seed) and statistics of the average network speed were collected. The required number of runs is determined based on the standard deviation of speed for a specific significance level and the tolerable error [35]. In this case, for $95 \%$ significance level and tolerable error equal to $0.5 \mathrm{~km} / \mathrm{h}$, the required number of runs was determined to be 10 .

\section{Results}

Simulation results were analyzed for the "do-nothing" (i.e. no dynamic eco-driving), VPA, and EVPA scenarios in three different aggregation levels:

- single vehicle performance (along different routes)

- average section statistics (along different IAs)
- average network-wide statistics (whole simulated network)

To facilitate the description of the results we use the capital letters assigned to different traffic lights of the test site (Fig. 1) to indicate road sections of interest in the context of this analysis. Specifically, the notation $\{\mathrm{R} \rightarrow \mathrm{Q}\}$ connotes the road section between traffic lights $\mathrm{R}$ and $\mathrm{Q}$. The arrow symbol determines the direction of traffic along the road section. In the cases of VPA and EVPA scenarios, it is also implied that dynamic ecodriving is deployed on the corresponding road section. The analysis of simulation results is presented in three dedicated subsections (per aggregation level) below.

\subsection{Single vehicle performance}

The analysis of single vehicle performance encompasses four different types of plots: a) speed vs distance, b) speed vs time, c) cumulative $\mathrm{CO}_{2}$ emissions vs distance, and d) acceleration vs speed. These plots reveal the influence of dynamic eco-driving on $\mathrm{CV}$ behavior and the corresponding $\mathrm{CV}$ performance in terms of $\mathrm{CO}_{2}$ emissions. The CV performance displayed in Figs. 2 and 3 pertains to traffic demand level D50, penetration rate P100 and two different routes of the test site.

Figure 2 shows information about a single CV performance along IA: $\{\mathrm{R} \rightarrow \mathrm{Q}\}$ (one-way multi-lane road segment). While the CV has to stop at the traffic light in the "do-nothing" scenario, it can adopt a deceleration strategy in the VPA and EVPA scenarios to avoid a standstill and generate lesser $\mathrm{CO}_{2}$ emissions. However, it can be seen (in the focus area of the right top plot) that the EVPA algorithm allows the CV to cruise at a marginally lower speed compared to the VPA one, and consequently begin acceleration approximately $10 \mathrm{~m}$ upstream of the traffic light (when the signal status changes to green).

As explained in Section 3 of this study, the latter behavior can promote comfort, safety and user acceptance of the system since the CV will not reach the traffic light (in red status) at cruising speed (enhanced speed advice); and increase intersection safety since there will be further available time for intersection clearance or $\mathrm{CV}$ tactical maneuvering in case of red light running from

Table $2 \mathrm{CO}_{2}$ emission constants per combination of vehicle and engine type

\begin{tabular}{llllllll}
\hline Vehicle Type & Engine Type & $\boldsymbol{E}_{\mathbf{0}}$ & $\boldsymbol{f}_{\mathbf{1}}$ & $\boldsymbol{f}_{\mathbf{2}}$ & $\boldsymbol{f}_{\mathbf{3}}$ & $\boldsymbol{f}_{\mathbf{4}}$ & \multicolumn{1}{l}{$\boldsymbol{f}_{\mathbf{5}}$} \\
\hline Car & Petrol & 0 & 0.553 & 0.161 & -0.003 & 0.266 & 0.511 \\
Car & Diesel & 0 & 0.324 & 0.086 & 0.005 & -0.059 & 0.448 \\
Car & LPG & 0 & 0.6 & 0.219 & -0.008 & 0.357 & 0.183 \\
Taxi & Diesel & 0 & 0.324 & 0.086 & 0.005 & -0.059 & 0.448 \\
HDV & Diesel & 0 & 1.52 & 1.88 & -0.07 & 4.71 & 0.17 \\
Bus & Diesel & 0 & 0.904 & 1.13 & -0.043 & 2.81 & 3.88 \\
\hline
\end{tabular}


Table 3 Simulated demand levels and penetration rates of the dynamic eco-driving service

\begin{tabular}{|c|c|c|c|c|c|c|c|c|}
\hline \multirow{2}{*}{$\begin{array}{l}\text { Demand Level } \\
\text { (D\%) }\end{array}$} & \multicolumn{8}{|c|}{ Penetration Rate $(\mathrm{P} \%)$} \\
\hline & $\mathrm{P} 0$ & P5 & $P 10$ & $P 15$ & $P 25$ & $P 50$ & P75 & $P 100$ \\
\hline D10 & $\checkmark$ & $x$ & $x$ & $\checkmark$ & $x$ & $\checkmark$ & $x$ & $\checkmark$ \\
\hline D20 & $\checkmark$ & $x$ & $x$ & $\checkmark$ & $x$ & $\checkmark$ & $x$ & $\checkmark$ \\
\hline D30 & $\checkmark$ & $x$ & $x$ & $\checkmark$ & $x$ & $\checkmark$ & $x$ & $\checkmark$ \\
\hline D40 & $\checkmark$ & $x$ & $x$ & $\checkmark$ & $x$ & $\checkmark$ & $x$ & $\checkmark$ \\
\hline D50 & $\checkmark$ & $\checkmark$ & $\checkmark$ & $\checkmark$ & $\checkmark$ & $\checkmark$ & $\checkmark$ & $\checkmark$ \\
\hline D60 & $\checkmark$ & $x$ & $x$ & $\checkmark$ & $x$ & $\checkmark$ & $x$ & $\checkmark$ \\
\hline D70 & $\checkmark$ & $x$ & $x$ & $\checkmark$ & $x$ & $\checkmark$ & $x$ & $\checkmark$ \\
\hline D80 & $\checkmark$ & $x$ & $x$ & $\checkmark$ & $x$ & $\checkmark$ & $x$ & $\checkmark$ \\
\hline D90 & $\checkmark$ & $x$ & $x$ & $\checkmark$ & $x$ & $\checkmark$ & $x$ & $\checkmark$ \\
\hline D100 & $\checkmark$ & $\checkmark$ & $\checkmark$ & $\checkmark$ & $\checkmark$ & $\checkmark$ & $\checkmark$ & $\checkmark$ \\
\hline
\end{tabular}
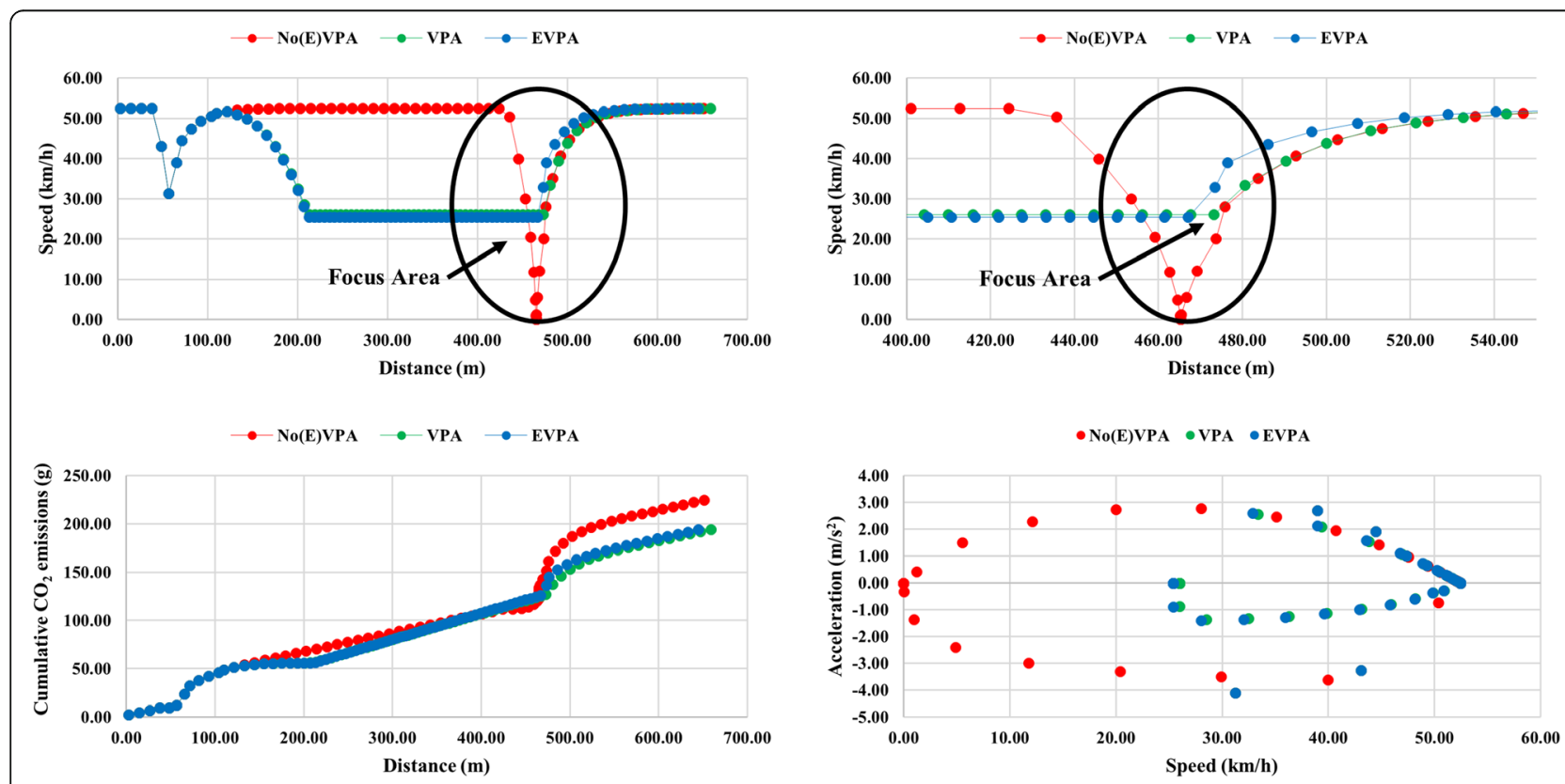

Fig. 2 Single vehicle performance on $I A:\{R \rightarrow Q\}$ 


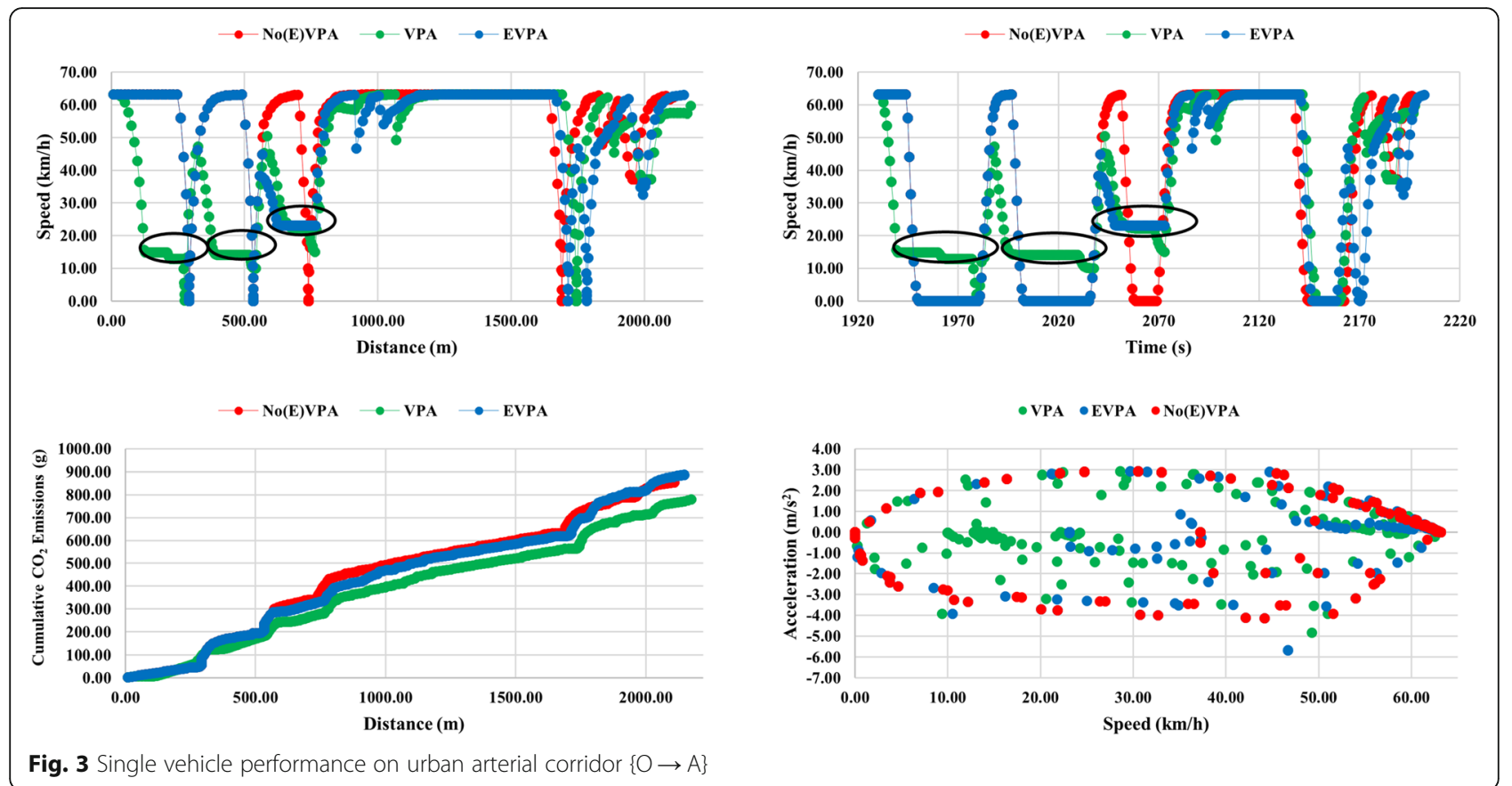

vehicles driving along other directions (possible scenario in mixed traffic conditions). Interestingly, the EVPA deceleration strategy does not adversely impact $\mathrm{CO}_{2}$ emissions savings. This is also justified by the same acceleration/deceleration patterns between VPA and EVPA depicted in Fig. 2 (bottom right plot).

The behavior of a single CV with (VPA and EVPA) and without ("do-nothing") dynamic eco-driving technology is examined along the urban arterial corridor $\{\mathrm{O} \rightarrow \mathrm{A}\}$. Every signalized intersection is equipped with a road-side unit (RSU) along the corridor (one-way fourlane urban arterial corridor with reserved bus lane on the right-most lane and side-street parking on the leftmost lane), thus enabling CVs to implement separate acceleration/deceleration strategies per IA.

Figure 3 (top plots) indicates that VPA allows the CV to successfully execute a deceleration strategy thrice, while EVPA only once given road characteristics, prevailing traffic conditions, and deployed traffic signal plan. However, the first two deceleration strategies suggested by VPA lead to rather low cruising speeds $(<20$ $\mathrm{km} / \mathrm{h}$ ) that can be non-acceptable by drivers or passengers in the case of fully autonomous vehicles. Moreover, they yield $\mathrm{CO}_{2}$ emissions savings that are not significant compared to the "do-nothing" and EVPA scenarios when the same CV has to fully stop at the traffic light and accelerate back to desired speed from standstill.

Nonetheless, a noteworthy observation is that dynamic eco-driving alters the traffic patterns of $\mathrm{CVs}$ even in space and time intervals that energy optimal driving strategies are not applied or possible. This phenomenon can generate unfavorable conditions for the $\mathrm{CV}$ due to surrounding traffic (queued vehicles disrupting the adoption of speed advice) or mistimed entrance at an intersection approach. Hence, the cumulative $\mathrm{CO}_{2}$ emissions of the CV (EVPA case) eventually surpass those of the unequipped equivalent (left bottom plot) along the examined path. Finally, results demonstrate that the VPA produces milder acceleration/deceleration rates for the examined $\mathrm{CV}$ (right bottom plot), and thus lesser cumulative $\mathrm{CO}_{2}$ emissions along its travelled path $\{\mathrm{O} \rightarrow \mathrm{A}\}$.

\subsection{Individual intersection approach statistics}

A plethora of information is provided to scrutinize the performance of dynamic eco-driving on two benchmark IAs of the test site and compare the behavior of VPA and EVPA methods. The evaluation of the different algorithms is conducted in terms of $\mathrm{CO}_{2}$ emissions $(\mathrm{gr} / \mathrm{km})$, number of stops per vehicle, and mean travel time (seconds). The reported travel time and $\mathrm{CO}_{2}$ emissions results also consider the road sections downstream of the examined IAs where benefits from energy efficient deceleration strategies can be realized. Moreover, a comprehensive analysis of the provided speed advice is presented per IA. Results are analyzed for traffic demand levels D50 (uncongested conditions) and D100 (congested conditions), and penetration rates ranging between P5 - P100. 
IA: $\{\mathrm{R} \rightarrow \mathrm{Q}\}$ was selected as benchmark in the context of this study since it is isolated and vehicle arrival patterns are not influenced by implementation of dynamic eco-driving along upstream IAs. Moreover, it is a oneway four-lane road section spanning up to $360 \mathrm{~m}$ where there is available space for CVs to adopt dynamic ecodriving maneuvers. SPaT messages are received up to $360 \mathrm{~m}$ upstream of signalized intersection $\mathrm{Q}$ by CVs, and $65.00 \mathrm{~s}$ of the signal cycle $(72.22 \%$ of the cycle duration) are allocated to the through movement (speed advice is estimated specifically for this movement). The minimum cruising speed is $20 \mathrm{~km} / \mathrm{h}$ in the case of EPVA, and $5 \mathrm{~km} / \mathrm{h}$ in the case of VPA. An influence zone calibration parameter of 0.01 indicates that CVs will reach the traffic signal on red light status while driving at cruising speed in the end of a deceleration strategy (VPA scenario). On the other hand, a 0.5 parameter value (EVPA scenario) ensures that CVs' acceleration will commence prior to arrival on red signal status to the intersection stop line.

Despite increased demand in D100, traffic conditions remain uncongested along IA: $\{\mathrm{R} \rightarrow \mathrm{Q}\}$ (Fig. 4). Mean travel time $(\mathrm{min} / \mathrm{km})$ is slightly affected by dynamic ecodriving (bottom plots) and mostly for higher penetration rates $(>75 \%)$. Both VPA and EVPA manage to significantly reduce idling (number of stops/veh) in mixed traffic, while stop events almost vanish in the case of fully equipped fleet (middle plots). However, it can be noticed that for low to intermediate penetration rates (P15 P50) and highest demand level (D100) EVPA outperforms VPA in terms of preventing CV stops at traffic light Q. VPA advices lower cruising speeds in the context of deceleration strategies, and thus non-equipped vehicles (which represent the highest share in the fleet mix for low penetration rates of dynamic eco-driving technology) tend to overpass CVs causing more stops at traffic lights compared to the EVPA scenario. Both algorithms generate $\mathrm{CO}_{2}$ emissions savings beyond medium penetration rate (P50) that are maximized for fully equipped fleet (P100). Maximum $\mathrm{CO}_{2}$ emissions savings rise approximately to $7.0 \%$ (top plots) and do not occur in the expense of significant travel time costs (bottom plots). Moreover, VPA and EVPA exhibit similar $\mathrm{CO}_{2}$ emissions savings potential in the case of IA: $\{R \rightarrow Q\}$.

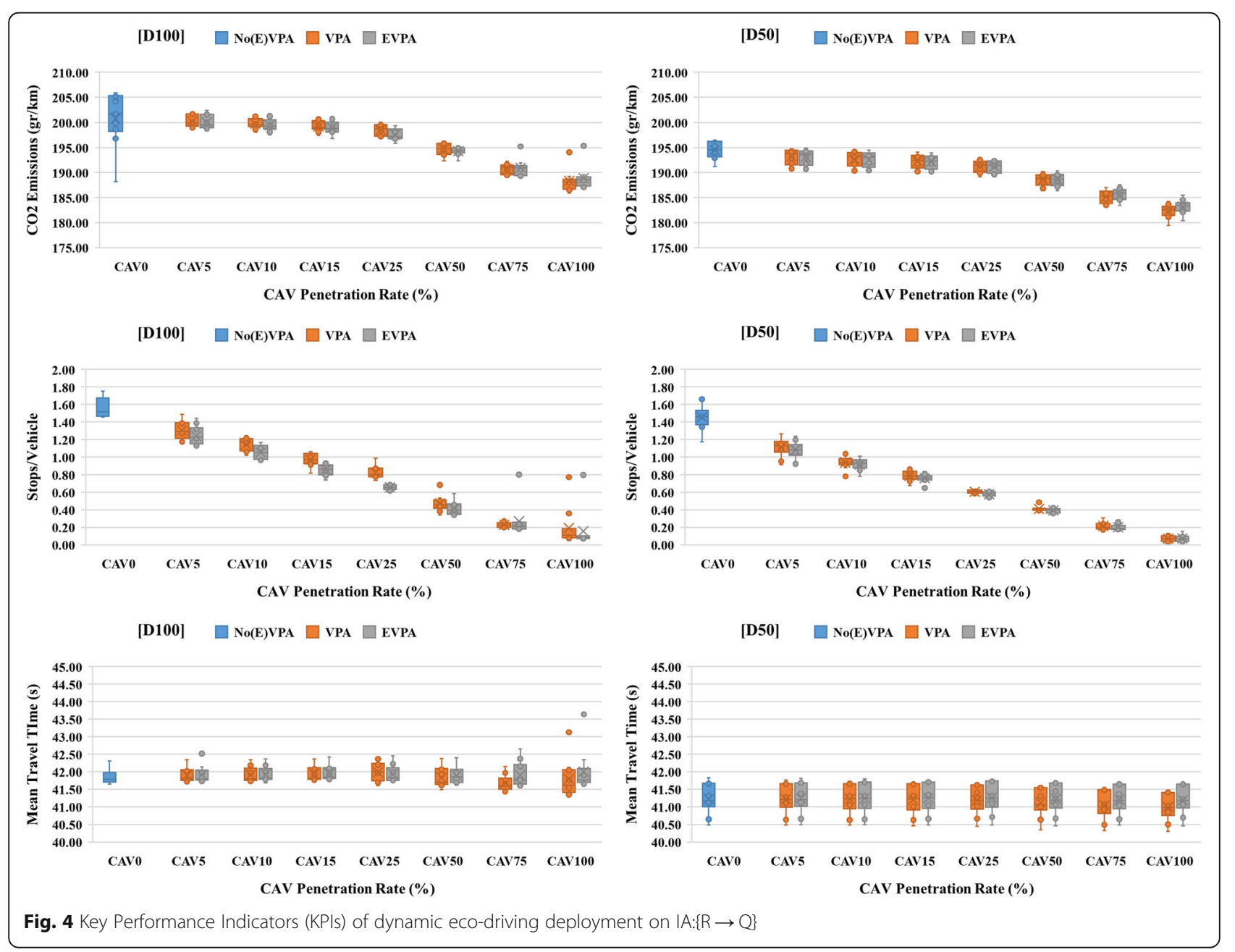


The reason IA: $\{N \rightarrow M\}$ is selected as benchmark and studied explicitly is multifold. IA: $\{N \rightarrow M\}$ is part of urban arterial corridor $\{\mathrm{O} \rightarrow \mathrm{A}\}$ where dynamic ecodriving is deployed on all signalized IAs (Fig. 1). It is one of the few IAs on urban arterial corridor $\{\mathrm{O} \rightarrow \mathrm{A}\}$ that spans $240 \mathrm{~m}$ long, thus providing enough space for CVs to execute dynamic eco-driving maneuvers. Additionally, it is fed with traffic by three different IAs (i.e. $\{\mathrm{O} \rightarrow \mathrm{N}\}$, $\{P \rightarrow N\}$, and $\{R \rightarrow N\}$ ) where dynamic eco-driving is also applied. Hence, vehicle arrival patterns vary significantly on IA: $\{\mathrm{N} \rightarrow \mathrm{M}\}$ giving the opportunity to test dynamic eco-driving for different $\mathrm{CV}$ approach speeds (also influenced upstream by dynamic eco-driving). Furthermore, $62.00 \mathrm{~s}$ of the signal cycle $(68.89 \%$ of the cycle duration) are allocated to the through movement (speed advice is estimated specifically for this movement). Consequently, there is adequate red duration to induce energy efficient deceleration strategies. Algorithmic settings (VPA and EVPA) for IA: $\{\mathrm{N} \rightarrow \mathrm{M}\}$ are similar to that of $\{\mathrm{R} \rightarrow \mathrm{Q}\}$.

Congested conditions prevail along IA: $\{\mathrm{N} \rightarrow \mathrm{M}\}$ for the highest demand level (D100). Mean travel time increases four times compared to uncongested conditions (D50) for the "do-nothing" scenario (Fig. 5). The deployment of dynamic eco-driving further disrupts traffic flow on IA: $\{N \rightarrow M\}$ for higher penetration rates. As explained in Section 3 of this study, both VPA and EVPA do not account for traffic light queues when estimating acceleration/deceleration strategies. Therefore, CVs can receive speed advice upon entrance to the intersection approach but eventually will need to abort it (due to reaching tail of queue), thus escalating travel time and $\mathrm{CO}_{2}$ emissions. Noticeably, EVPA outperforms VPA on the basis of the examined KPIs (left plots - D100) for the majority of the tested penetration rates (most significant difference for higher penetration rates). Due to the higher minimum speed advice threshold in the case of EVPA (i.e. $20 \mathrm{~km} / \mathrm{h}$ ), lesser speed advices are provided to equipped vehicles, hence reducing the intensity of disruption to the traffic flow and $\mathrm{CO}_{2}$ emissions performance incurred by dynamic eco-driving.

On the other hand, traffic conditions are uncongested along IA: $\{\mathrm{N} \rightarrow \mathrm{M}\}$ for the intermediate demand scenario (D50). Queued traffic almost diminishes at traffic light $\mathrm{M}$ (Fig. 5 - middle right plot) for higher penetration rates $(>75 \%)$. EVPA generates $\mathrm{CO}_{2}$ emissions savings along IA: $\{\mathrm{N} \rightarrow \mathrm{M}\}$, which approximately rise to $13.0 \%$ and $8.5 \%$ reduction compared to the "do-nothing" and VPA scenarios respectively (Fig. 5 - top right plot). Notably, EVPA exhibits significantly improved performance compared to VPA with respect to emissions reduction, although it adapts speed advice to improve comfort and safety. Finally, it can be observed that for low to intermediate penetration rates vehicle stops increase with deployment of VPA. This phenomenon occurs due to the behavior of non-equipped vehicles as it was explained in the aforementioned analysis of simulation results for IA: $\{\mathrm{R} \rightarrow \mathrm{Q}\}$ as well.

\subsection{Network-wide statistics}

The effects of dynamic eco-driving (VPA and EVPA) on network performance are assessed in terms of: i) average network speed, b) $\mathrm{CO}_{2}$ emissions per kilometer driven $(\mathrm{gr} / \mathrm{km})$, and c) average stop time per kilometer driven $(\mathrm{s} / \mathrm{km})$. Network-wide statistics are reported for the full spectrum of examined demand levels (D10 - D100) and two penetration rates (P50 and P100) to identify triggering points for VPA and EVPA activation according to the prevailing traffic conditions on the examined test site.

Figure 6 indicates that both VPA and EVPA can yield $\mathrm{CO}_{2}$ emissions savings when average network speed is over $25 \mathrm{~km} / \mathrm{h}(\mathrm{D} 10$ - D80), but the latter savings are insignificant though. Moreover, network-wide savings diminish as traffic demand shifts from light to moderate $(\mathrm{D} 10 \rightarrow \mathrm{D} 80)$. On the other hand, the tested algorithms exhibit similar performance to the "do-nothing" case for heavy traffic conditions (congestion) when average stop time increases significantly both for $50 \%$ and $100 \%$ penetration rates. As aforementioned, this is reasonable considering that both algorithms are not designed to account for traffic light queues when estimating energy efficient speed advice. Moreover, it can be seen that lower share of CVs in the fleet mix (P50) results in slightly lesser impacts of dynamic eco-driving on the network scale compared to the case of fully equipped fleet (P100).

VPA generates marginally higher $\mathrm{CO}_{2}$ emissions savings compared to EVPA in uncongested conditions when the whole test site is considered. However, these savings are realized in the expense of marginally increased travel times. Longer travel times are expected in the VPA scenarios due to the minimum speed advice threshold (i.e. $5 \mathrm{~km} / \mathrm{h}$ ). Lower $\mathrm{CO}_{2}$ emissions on the network level can be attributed to more energy efficient patterns generated by VPA at areas of the network where speed advice is not implemented successfully or at all as previously highlighted and explained in the analysis of single vehicle performance. Finally, the lower stop times observed for VPA can be also ascribed to crawling speeds that can be advised by the latter algorithm.

\subsection{Discussion}

Simulation results indicate that EVPA can exhibit similar or even better performance compared to VPA for specific road characteristics, activation distances of dynamic eco-driving service, traffic conditions and traffic signal 


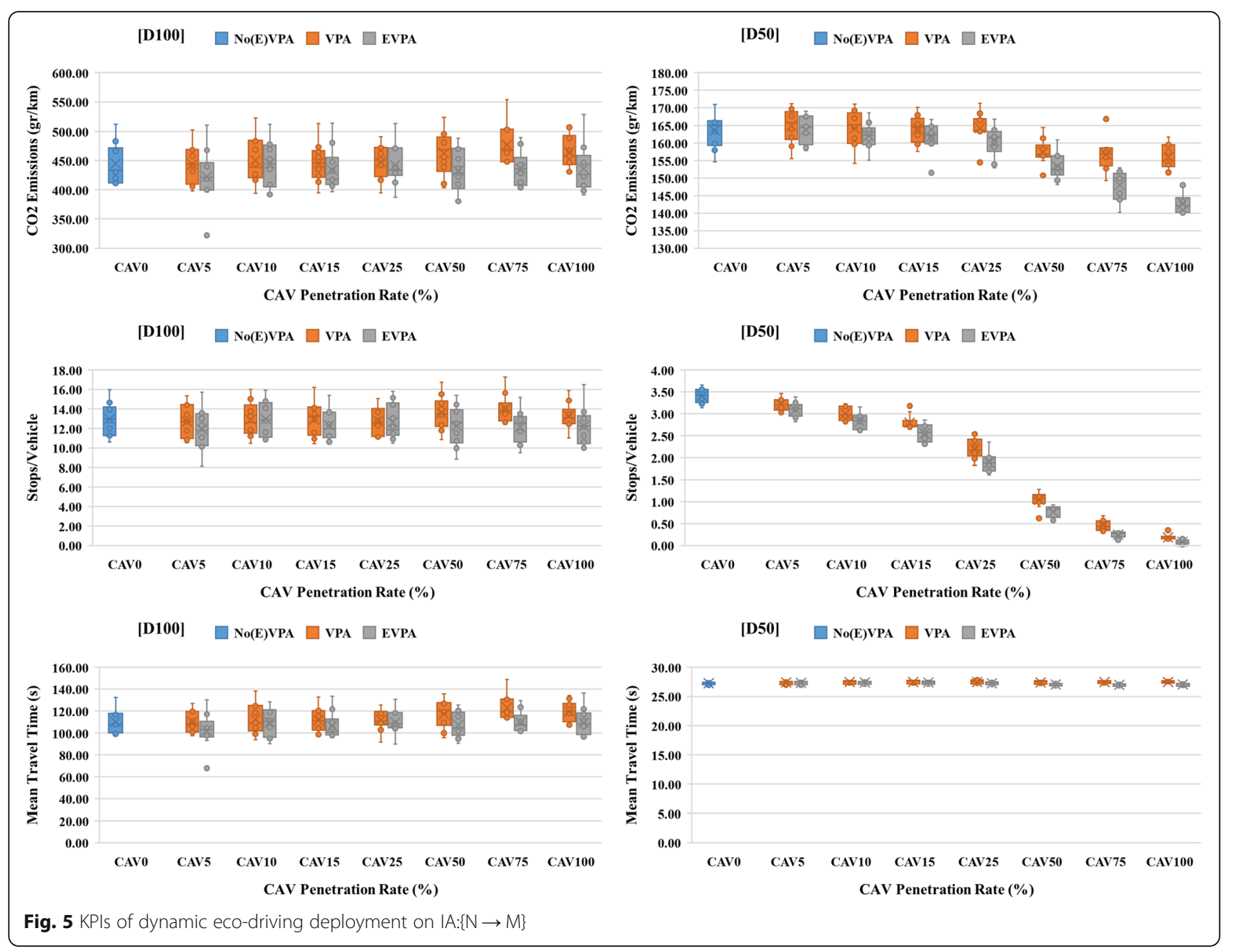

plans, despite adapting speed advice to improve user acceptance and intersection safety. It is also noteworthy, that improved EVPA performance occurs when VPA advices deceleration strategies that encompass cruising speeds that undercut the minimum cruising speed after deceleration $\left(v_{\text {min }}\right)$. For this reason EVPA and VPA performance is similar along IA: $\{\mathrm{R} \rightarrow \mathrm{Q}\}$, while EVPA significantly outperforms VPA in the case of IA: $\{N \rightarrow M\}$. Moreover, the fact that EVPA suggests vehicle acceleration prior to $\mathrm{CV}$ arrival at the signalized intersection after deceleration strategies does not weaken its ability to yield $\mathrm{CO}_{2}$ emissions savings.

On the other hand, the analysis of single vehicle performance and network-wide statistics revealed that VPA slightly outperforms EVPA in terms of environmental benefits on the network level. Nonetheless, this occurs at the cost of marginally higher travel times. Both VPA and EVPA generate different traffic patterns on the examined test site even in areas where speed advice is not feasible due to surrounding traffic or mistimed arrival at intersection approach.
Moreover, both algorithms do not produce significant network-wide emissions savings compared to the "donothing" scenario even for low to moderate traffic demand. As it can be seen in Fig. 1, traffic lights are closely spaced beyond traffic light $M$ along the urban arterial corridor $\{\mathrm{O} \rightarrow \mathrm{A}\}$, where the speed limit is $50 \mathrm{~km} / \mathrm{h}$ along $\{\mathrm{O} \rightarrow \mathrm{A}\}$. Thus, dynamic eco-driving benefits diminish due to low approach speeds, confined speed range and space for adapting to speed advice, and VPA/ EVPA algorithmic logic that considers single signalized intersections for estimating energy efficient driving strategies instead of multi-intersection corridors controlled by traffic lights. Previous research has also indicated that inappropriate deployment of dynamic eco-driving could even generate environmental disbenefits due to unfavorable factors [39, 40, 46, 55]. Hence, the deployment scheme of dynamic eco-driving that encompasses road design characteristics, activation distance of the service, traffic signal plans and traffic conditions significantly affect its energy efficiency and emissions savings potential. 


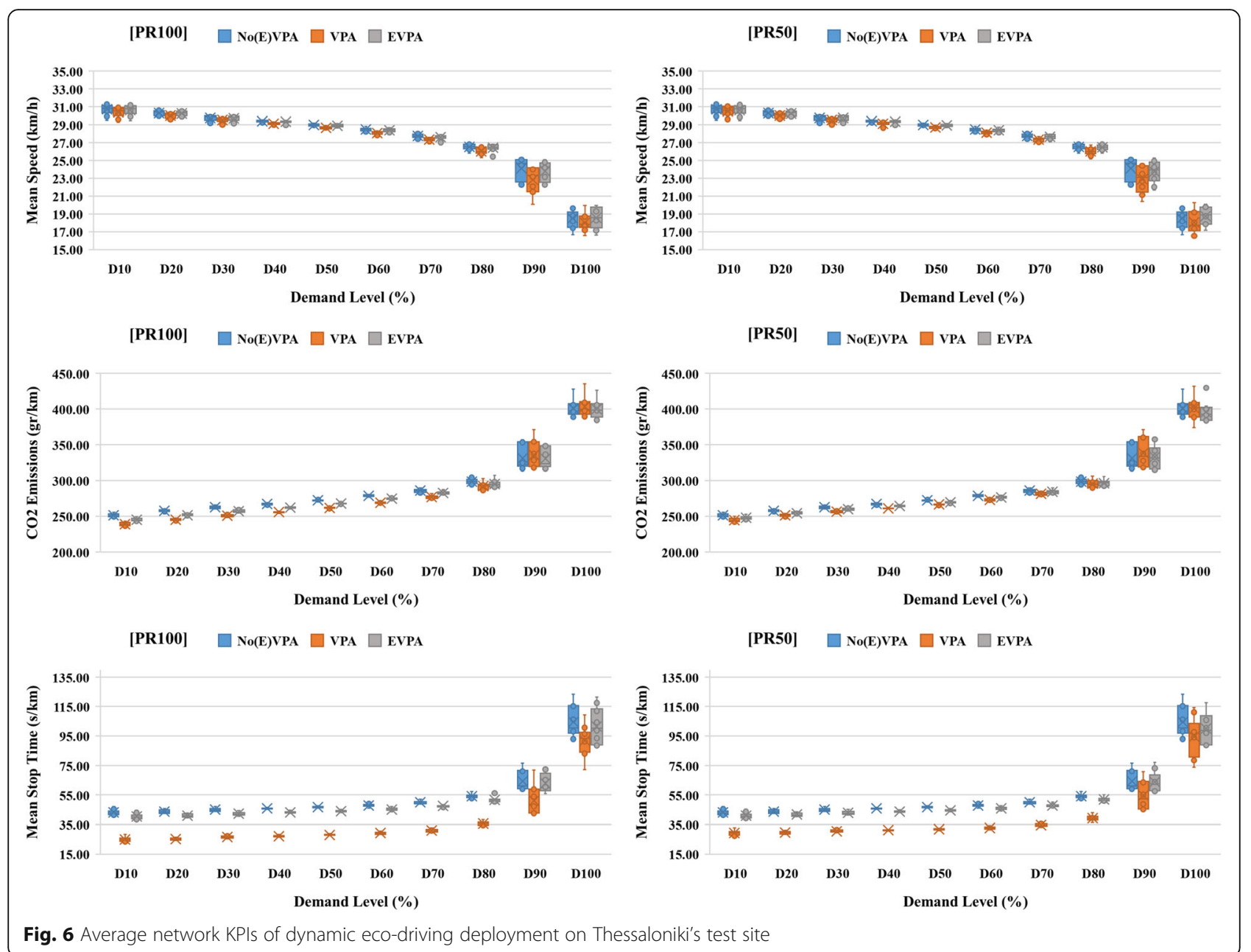

According to the latter information, it is important to identify the deployment scheme that enables EVPA to perform efficiently (in terms of $\mathrm{CO}_{2}$ emissions reduction) on the network scale. Thus, travel time, user acceptance and safety benefits also provided by EVPA can be realized.

Additionally, we show that VPA and EVPA deteriorate traffic conditions during congestion since they do not consider traffic light queues for speed advice estimation. The corresponding simulation results pose irregular patterns with respect to speed advice efficiency and $\mathrm{CO}_{2}$ emissions. Notably, interactions between CVs and nonequipped vehicles become more complex especially in the case of VPA when crawling speeds can be advised to CVs.

Finally, it is of note that we assumed full diver compliance to speed advice in the context of this simulation study. However, human factors can exert significant impacts on traffic flow performance [34] and intersection safety $[17,19]$. Hence, we plan to address partial compliance to speed advice based on real-world data in future research efforts and assess safety implications of human factors with respect to dynamic eco-driving with the use of rigorous mathematical methods such as the Surrogate Safety Assessment Model (SSAM).

\section{Conclusions}

$\mathrm{CV}$ applications have received significant attention from the research community in the past two decades. Several dynamic eco-driving models were proposed for the estimation of energy efficient speed advice in the vicinity of signalized intersections. This study focused on the enhancement of speed advice comfort and safety without negatively affecting energy and traffic efficiency. A microscopic simulation analysis on an actual urban network is conducted to evaluate the performance of an enhanced velocity planning algorithm (EVPA) for different penetration rates of dynamic eco-driving technology and traffic demand levels.

Simulation results indicated that EVPA can generate $\mathrm{CO}_{2}$ emissions savings on the order of $13 \%$ along individual intersection approaches and $2.5 \%$ on a network 
scale, without substantially escalating travel times. Moreover, EVPA ensures increased speed advice comfort and safety due to its inherent control logic. However, it was also identified that EVPA's efficiency is dependent on roadway characteristics, activation distance of the service, traffic signal plans and traffic conditions. Thus, the deployment scheme of dynamic eco-driving on urban networks plays a significant role in warranting environmental benefits and traffic efficiency.

Additionally, it was proven that speed advice estimation should consider signal plans from consecutive traffic lights on urban arterial corridors with closely spaced signalized intersections to increase dynamic eco-driving performance. Finally, this study explicitly assumed connected vehicles that can precisely follow speed advice using automation functions. In future research, the authors plan to utilize actual speed advice data to model the influence of human factors in the adoption of speed advice when CVs are manually driven and meticulously evaluate respective impacts on traffic safety and emissions savings.

\section{Acknowledgements}

Not applicable.

\section{Authors' contributions}

The author(s) read and approved the final manuscript.

\section{Funding}

This work was supported by the State Scholarships Foundation (IKY) under the IKY/SIEMENS Research Projects for Excellence Programme [grant number 1516] "Analysis and Upgrade of the Operations of Cooperative Intelligent Transportations Systems in Urban Networks".

\section{Availability of data and materials}

The datasets used and/or analysed during the current study are available from the corresponding author on reasonable request.

\section{Competing interests}

The authors declare that they have no competing interests.

\section{Author details}

'Department of Transportation Planning and Engineering, School of Civil Engineering, National Technical University of Athens, 5 Iroon Polytechniou Str., Zografou Campus, 15773 Athens, Greece. ${ }^{2}$ Centre for Research and Technology Hellas, Hellenic Institute of Transport, 6th km Charilaou-Thermi Rd, 57001 Thermi, Greece. ${ }^{3}$ MUMA College of Business, University of South Florida, 4202 E. Fowler Ave., BSN 3108, Tampa, FL 33620, USA.

Received: 3 March 2020 Accepted: 17 November 2020

Published online: 04 January 2021

\section{References}

1. ACEA. (2017). Vehicles in use - Europe 2017. European Automobile Manufacturers Association. https://www.acea.be/uploads/statistic documents/ACEA_Report_Vehicles_in_use-Europe_2017.pdf

2. Alam, M. S., \& McNabola, A. (2014). A critical review and assessment of ecodriving policy \& technology: Benefits \& limitations. Transport Policy, 35, 4249. https://doi.org/10.1016/.jtranpol.2014.05.016.

3. Archer, J. (2005). Indicators for traffic safety assessment and prediction and their application in micro-simulation modelling: A study of urban and suburban intersections. Doctoral Thesis, KTH.

4. Asadi, B., \& Vahidi, A. (2011). Predictive cruise control: Utilizing upcoming traffic signal information for improving fuel economy and reducing trip time. IEEE Transactions on Control Systems Technology, 19(3), 707-714. https://doi.org/10.1109/TCST.2010.2047860.

5. Barth, M., Mandava, S., Boriboonsomsin, K., \& Xia, H. (2011). Dynamic ECOdriving for arterial corridors. In In 2011 IEEE Forum on Integrated and Sustainable Transportation Systems (FISTS), (pp. 182-188). https://doi.org/10. 1109/FISTS.2011.5973594.

6. Bodenheimer, R., Brauer, A., Eckhoff, D., \& German, R. (2014). Enabling GLOSA for adaptive traffic lights. In 2014 IEEE Vehicular Networking Conference (VNC), (pp. 167-174). https://doi.org/10.1109/NNC.2014.7013336.

7. Chen, W., Liu, Y., Yang, X., Bai, Y., Gao, Y., \& Li, P. (2015). Platoon-based speed control algorithm for Ecodriving at signalized intersection. Transportation Res Rec, 2489(1), 29-38. https://doi.org/10.3141/2489-04.

8. Chu, L., Liu, H. X., Oh, J. S., \& Recker, W. (2003). A calibration procedure for microscopic traffic simulation. In Proceedings of the 2003 IEEE International Conference on Intelligent Transportation Systems (ITSC), (pp. 1574-1579). https://doi.org/10.1109/ITSC.2003.1252749.

9. Dalla Chiara, B., Deflorio, F. P., \& Cuzzola, S. (2014). A proposal of risk indexes at signalised intersections for ADAS aimed to road safety. In Informatics in Control, Automation and Robotics, (pp. 265-278). Cham: Springer. https://doi. org/10.1007/978-3-319-03500-0_17.

10. Dalla Chiara, B., Deflorio, F., \& Diwan, S. (2009). Assessing the effects of intervehicle communication systems on road safety. IET Intelligent Transport Systems, 3(2), 225-235. https://doi.org/10.1049/iet-its:20080059.

11. Erdmann, J. (2013). Combining adaptive junction control with simultaneous green-light-optimal-speed-advisory. In 2013 IEEE 5th International Symposium on Wireless Vehicular Communications (WiVeC), (pp. 1-5). https://doi.org/10. 1109/wivec.2013.6698230.

12. Gettman, D., \& Pu, L. (2006). Theoretical validation of surrogate safety assessment methodology for roundabouts and cross intersections. In Proceedings of the 13th ITS World Congress.

13. Guanetti, J., Kim, Y., \& Borrelli, F. (2018). Control of connected and automated vehicles: State of the art and future challenges. Annual Reviews in Control, 45, 18-40. https://doi.org/10.1016/j.arcontrol.2018.04.011.

14. Hao, P., Wu, G., Boriboonsomsin, K., \& Barth, M. J. (2018). Eco-approach and departure (EAD) application for actuated signals in real-world traffic. IEEE Transactions on Intelligent Transportation Systems, 20(1), 1-11. https://doi. org/10.1109/TITS.2018.2794509.

15. He, X., Liu, H. X., \& Liu, X. (2015). Optimal vehicle speed trajectory on a signalized arterial with consideration of queue. Transportation Research Part C: Emerging Technologies, 61, 106-120. https://doi.org/10.1016/j.trc.2015.11. 001.

16. Huang, Y., Ng, E. C. Y., Zhou, J. L., Surawski, N. C., Chan, E. F. C., \& Hong, G. (2018). Eco-driving technology for sustainable road transport: A review. Renewable and Sustainable Energy Reviews, 93, 596-609. https://doi.org/10. 1016/j.rser.2018.05.030.

17. Hurwitz, D. S. (2009). Application of driver behavior and comprehension to dilemma zone definition and evaluation. Doctoral Thesis, University of Massachusetts Amherst. https://doi.org/10.7275/bxwp-8c84.

18. Jiang, H., Hu, J., An, S., Wang, M., \& Park, B. B. (2017). Eco approaching at an isolated signalized intersection under partially connected and automated vehicles environment. Transportation Research Part C: Emerging Technologies, 79, 290-307. https://doi.org/10.1016/j.trc.2017.04.001.

19. Johansson, G., \& Rumar, K. (1971). Drivers' brake reaction times. Human factors, 13(1), 23-27. https://doi.org/10.1177/2F001872087101300104.

20. Kamalanathsharma, R. K., \& Rakha, H. A. (2016). Leveraging connected vehicle technology and telematics to enhance vehicle fuel efficiency in the vicinity of signalized intersections. Journal of Intelligent Transportation Systems, 20(1), 33-44. https://doi.org/10.1080/15472450.2014.889916.

21. Kamalanathsharma, R. K., Rakha, H. A., \& Yang, H. (2015). Networkwide impacts of vehicle Ecospeed control in the vicinity of traffic signalized intersections. Transportation Research Record: Journal of the Transportation Research Board, 2503(1), 91-99. https://doi.org/10.3141/2503-10.

22. Kim, K. J., \& Sul, J. (2009). Development of intersection traffic accident risk assessment model. In 4th IRTAD Conference.

23. Liang, X., Guler, S. I., \& Gayah, V. V. (2019). Joint optimization of signal phasing and timing and vehicle speed guidance in a connected and autonomous vehicle environment. Transportation Research Record: Journal of the Transportation Research Board, 2673(4), 70-83. https://doi.org/10.1177/ 0361198119841285.

24. Liao, R., Chen, X., Yu, L., \& Sun, X. (2018). Analysis of emission effects related to drivers' compliance rates for cooperative vehicle-infrastructure system at 
signalized intersections. International Journal of Environmental Research and Public Health, 15(1), 122. https://doi.org/10.3390/ijerph15010122.

25. Mahler, G., \& Vahidi, A. (2012). Reducing idling at red lights based on probabilistic prediction of traffic signal timings. In 2012 American Control Conference (ACC), (pp. 6557-6562). https://doi.org/10.1109/ACC.2012. 6314942.

26. Maile, M., \& Delgrossi, L. (2009). Cooperative intersection collision avoidance system for violations (cicas-v) for prevention of violation-based intersection crashes. In Proceedings of International Technical Conference on the Enhanced Safety of Vehicles.

27. Mandava, S., Boriboonsomsin, K., \& Barth, M. (2009). Arterial velocity planning based on traffic signal information under light traffic conditions. In 12th International IEEE Conference on Intelligent Transportation Systems (ITSC 2009), (pp. 1-6). https://doi.org/10.1109/ITSC.2009.5309519.

28. Mannering, F., Kilareski, W., \& Washburn, S. (2007). Principles of highway engineering and traffic analysis. USA: Wiley.

29. Mintsis, E., Vlahogianni, E. I., \& Mitsakis, E. (2020). Dynamic eco-driving near signalized intersections: Systematic review and future research directions. Journal of Transportation Engineering, Part A: Systems, 146(4). https://doi.org/ 10.1061/JTEPBS.0000318.

30. Monteil, J., Billot, R., \& El Faouzi, N. E. (2011). Towards cooperative traffic management: methodological issues and perspectives. In Proceedings of Australasian Transport Research Forum, (pp. 28-30).

31. Morsink, P. L., Wismans, L. J., \& Dijkstra, A. (2008). Micro-simulation for road safety impact assessment of advanced driver assistance systems. In 7th European Congress and Exhibition on Intelligent Transport Systems and Services.

32. Mousa, S. R., Ishak, S., Mousa, R. M., \& Codjoe, J. (2019). Developing an ecodriving application for semi-actuated signalized intersections and modeling the market penetration rates of eco-driving. Transportation Research Record: Journal of the Transportation Research Board, 2673(5), 466-477. https://doi. org/10.1177/0361198119839960.

33. Mousa, S. R., Mousa, R., \& Ishak, S. (2018). A Deep-Reinforcement Learning Algorithm for Eco-Driving Control at Signalized Intersections with Prioritized Experience Replay, Target Network, and Double Learning. In Transportation Research Board 97th Annual Meeting https://trid.trb.org/view/1497121.

34. Ni, D., Li, L., Wang, H., \& Jia, C. (2017). Observations on the fundamental diagram and their interpretation from the human factors perspective. Transportmetrica B: transport dynamics, 5(2), 159-176. https://doi.org/10. 1080/21680566.2016.1190673.

35. Ott, L., \& Longnecker, M. (2004). A first course in statistical methods. Thomson-Brooks/Cole.

36. Panis, L. I., Broekx, S., \& Liu, R. (2006). Modelling instantaneous traffic emission and the influence of traffic speed limits. Science of The Total Environment, 371(1-3), 270-285. https://doi.org/10.1016/j.scitotenv.2006.08. 017.

37. Pipes, L. A. (1953). An operational analysis of traffic dynamics. Journal of Applied Physics, 24(3), 274-281. https://doi.org/10.1063/1.1721265.

38. Qi, X., Wang, P., Wu, G., Boriboonsomsin, K., \& Barth, M. J. (2018). Connected cooperative Ecodriving system considering human driver error. IEEE Transactions on Intelligent Transportation Systems, 19(8), 2721-2733. https:// doi.org/10.1109/TITS.2018.2845799.

39. Rakha, H. A., Chen, H., Almannaa, M., El-Shawarby, I., \& Loulizi, A. (2016). Developing and field implementing an Ecocruise control system in the vicinity of traffic signalized intersections (no. NY17-02). TranLIVE. University of Idaho https://rosap.ntl.bts.gov/view/dot/36797.

40. Rakha, H. A., Kamalanathsharma, R. K., \& Ahn, K. (2012). AERIS: Eco-vehicle speed control at signalized intersections using I2V communication (FHWA-JPO12-063). Virginia Tech Transportation Institute http://ntl.bts.gov/lib/46 000/46300/46329/FHWA-JPO-12-063_FINAL_PKG.pdf

41. Raubitschek, C., Schutze, N., Kozlov, E., \& Baker, B. (2011). Predictive driving strategies under urban conditions for reducing fuel consumption based on vehicle environment information. In 2011 IEEE Forum on Integrated and Sustainable Transportation Systems (FISTS), (pp. 13-19). https://doi.org/10. 1109/FISTS.2011.5973609.

42. Schuricht, P., Michler, O., \& Bäker, B. (2011). Efficiency-increasing driver assistance at signalized intersections using predictive traffic state estimation. In 14th International IEEE Conference on Intelligent Transportation Systems (ITSC 2011), (pp. 347-352). https://doi.org/10.1109/ITSC.2011.6083111.

43. Stebbins, S., Hickman, M., Kim, J., \& Vu, H. L. (2017). Characterising green light optimal speed advisory trajectories for platoon-based optimisation.
Transportation Research Part C: Emerging Technologies, 82, 43-62. https://doi. org/10.1016/j.trc.2017.06.014

44. Sun, C., Guanetti, J., Borrelli, F., \& Moura, S. (2018). Robust eco-driving control of autonomous vehicles connected to traffic lights. ArXiv: 1802.05815 [Math]. http://arxiv.org/abs/1802.05815

45. Taiebat, M., Brown, A. L., Safford, H. R., Qu, S., \& Xu, M. (2018). A review on energy, environmental, and sustainability implications of connected and automated vehicles. Environmental Science \& Technology, 52(20), 11449 11465. https://doi.org/10.1021/acs.est.8b00127.

46. Tielert, T., Killat, M., Hartenstein, H., Luz, R., Hausberger, S., \& Benz, T. (2010). The impact of traffic-light-to-vehicle communication on fuel consumption and emissions. In 2010 Internet of Things (IOT), (pp. 1-8). https://doi.org/10.1109/ IOT.2010.5678454

47. Vahidi, A., \& Sciarretta, A. (2018). Energy saving potentials of connected and automated vehicles. Transportation Research Part C: Emerging Technologies, 95, 822-843. https://doi.org/10.1016/j.trc.2018.09.001.

48. Vreeswijk, J. D., Mahmod, M. K. M., \& van Arem, B. (2010). Energy efficient traffic management and control - The eCoMove approach and expected benefits. In 13th International IEEE Conference on Intelligent Transportation Systems (ITSC 2010), (pp. 955-961). https://doi.org/10.1109/ITSC.2010. 5625122.

49. Wadud, Z., MacKenzie, D., \& Leiby, P. (2016). Help or hindrance? The travel, energy and carbon impacts of highly automated vehicles. Transportation Research Part A: Policy and Practice, 86, 1-18. https://doi.org/10.1016/j.tra. 2015.12.001

50. Wan, N., Vahidi, A., \& Luckow, A. (2016). Optimal speed advisory for connected vehicles in arterial roads and the impact on mixed traffic. Transportation Research Part C: Emerging Technologies, 69, 548-563. https:// doi.org/10.1016/j.trc.2016.01.011.

51. Wang, Z., Wu, G., \& Barth, M. J. (2019). Cooperative eco-driving at signalized intersections in a partially connected and automated vehicle environment. In IEEE Transactions on Intelligent Transportation Systems, (pp. 1-10). https:// doi.org/10.1109/TITS.2019.2911607.

52. Wang, Z., Wu, G., Hao, P., \& Barth, M. J. (2018). Cluster-wise cooperative ecoapproach and departure application for connected and automated vehicles along signalized arterials. IEEE Transactions on Intelligent Vehicles, 3(4), 404413. https://doi.org/10.1109/TIV.2018.2873912

53. Weber, A., \& Winckler, A. (2013). Exploratory advanced research project: Advanced traffic signal control. BMW Final Report. https://merritt.cdlib.org/ d/ark:\%2F13030\%2Fm5s4865n/1/producer\%2F884614370.pdf

54. Wu, X., Zhao, X., Xin, Q., Yang, Q., Yu, S., \& Sun, K. (2019). Dynamic cooperative speed optimization at signalized arterials with various platoons. Transportation Research Record: Journal of the Transportation Research Board, 2673(5), 528-537. https://doi.org/10.1177/0361198119839964.

55. Xia, H., Boriboonsomsin, K., \& Barth, M. (2013). Dynamic eco-driving for signalized arterial corridors and its indirect network-wide energy/emissions benefits. Journal of Intelligent Transportation Systems, 17(1), 31-41. https:// doi.org/10.1080/15472450.2012.712494.

56. Xia, H., Wu, G., Boriboonsomsin, K., \& Barth, M. (2013). Development and evaluation of an enhanced eco-approach traffic signal application for connected vehicles. In 16th International IEEE Conference on Intelligent Transportation Systems (ITSC 2013), (pp. 296-301). https://doi.org/10.1109/ ITSC.2013.6728248

57. Xiang, X., Zhou, K., Zhang, W.-B., Qin, W., \& Mao, Q. (2015). A closed-loop speed advisory model with Driver's behavior adaptability for eco-driving. IEEE Transactions on Intelligent Transportation Systems, 16(6), 3313-3324. https://doi.org/10.1109/TITS.2015.2443980.

58. Yang, Z., Zeng, H., Yu, Z., Wei, X., Liu, A., \& Fan, X. (2019). Research on ecodriving strategy at intersection based on vehicle infrastructure cooperative system. Advances in Mechanical Engineering, 11(4). https://doi.org/10.1177/ 1687814019843368.

59. Zhou, F., Li, X., \& Ma, J. (2017). Parsimonious shooting heuristic for trajectory design of connected automated traffic part I: Theoretical analysis with generalized time geography. Transportation Research Part B: Methodological, 95, 394-420. https://doi.org/10.1016/j.trb.2016.05.007.

\section{Publisher's Note}

Springer Nature remains neutral with regard to jurisdictional claims in published maps and institutional affiliations. 